\title{
Independent Gradient Model: a new approach for probing strong and weak interactions in molecules from wave function calculations
}

\author{
Corentin Lefebvre, ${ }^{[\mathrm{a}]}$ Hassan Khartabil, ${ }^{[\mathrm{a}, \mathrm{b}]}$ Jean-Charles Boisson, ${ }^{[\mathrm{c}]}$ Julia Contreras-García, ${ }^{[\mathrm{d}]}$ Jean- \\ Philip Piquemal ${ }^{[\mathrm{d}]}$ and Eric Hénon ${ }^{*}[\mathrm{a}]$
}

\begin{abstract}
Extracting the chemical interaction signature from local descriptors based on electron density (ED) is still a fruitful field of development in chemical interpretation. In a previous work using promolecular ED (frozen ED), the new descriptor $\delta g$ was defined. It represents the difference between a virtual upper limit of the ED gradient $\left(\left|\nabla \rho^{I G M}\right|\right)$ representing a non-interacting system and the true ED gradient $|\nabla \rho|$. It can be seen as a measure of electron sharing brought by ED contragradience. A compelling feature of this model is to provide an automatic workflow that extracts the signature of interactions between selected groups of atoms. As with the $\mathrm{NCl}$ (Non Covalent Interaction) approach, it provides chemists with a visual understanding of interactions present in chemical systems. $\left|\nabla \rho^{I G M}\right|$ is achieved simply by using absolute values upon summing the individual gradient contributions making up the total ED gradient. Hereby, we extend this model to relaxed ED calculated from a wave function. To this end, we formulate the Gradient-Based Partitioning (GBP) to assess the contribution of each orbital to the total ED gradient. We highlight these new possibilities across two prototypical examples of organic chemistry: the unconventional hexamethylbenzene dication involving a hexa-coordinated carbon atom and the $\beta$-thioaminoacrolein. It will be shown how a bond-bybond picture can be obtained from a wave function opening the way to monitor specific interactions along reaction paths.
\end{abstract}

\section{Introduction}

A number of tools have been derived from the analysis of the

[a] PhD, C. Lefebvre, Dr H. Khartabil, Pr E. Hénon Institut de Chimie Moléculaire de Reims UMR CNRS 7312 University of Reims Champagne-Ardenne

Moulin de la Housse 51687 Reims Cedex 2 BP 39 (France)

E-mail: eric.henon@univ-reims.fr

[b] Dr H. Khartabil

Campus Universitaire des Ardennes

University of Reims Champagne-Ardenne

4 Bd Jean Delautre, 08000 Charleville-Mézières (France)

[c] Dr J-C. Boisson

CReSTIC EA 3804

University of Reims Champagne-Ardenne

Moulin de la Housse 51687 Reims Cedex 2 BP 39 (France)

[d] Dr J. Contreras-García, Pr J.-P. Piquemal

Sorbonne Universités, UPMC, Laboratoire de Chimie Théorique and CNRS UMR CNRS 7616

4 PI Jussieu, 75252 Paris Cedex 05 (France)

Supporting information for this article is given via a link at the end of the document. electron density $\rho$ in a molecular system, e.g. QTAIM ${ }^{[1]}$ (Quantum Theory of Atoms in Molecules), ELF ${ }^{[2]}$ (Electron Localization Function) and LED ${ }^{[3]}$ (Localized Electron Detector). Such techniques are useful for the interpretation of quantum chemical computations by extracting pieces of information that may be connected to simple concepts used by chemists such as atoms linked by bonds. The implementation of such analysis may also provide a valuable theoretical support to challenging experimental electronic structures. The $\mathrm{NCI}^{[4]}$ approach, another local ED-based descriptor developed by one of the present authors, is one of the modern tools for chemical bonding analysis. It is exclusively given in terms of electron density $\rho$ (ED) and its gradient. Although $\mathrm{NCl}$ is able to reveal covalent bonding pattern, ${ }^{[5]}$ it is usually devoted to the visualization of weak interactions where previous tools were conspicuously failing. This approach provides a direct three-dimensional representation in the form of closed domains that highlight the spatial localization of the interactions within the chemical system. In these pictures, through the application of the $\mathrm{NCl}$ index, the nature of the interaction can be visually associated in practice to a color code directly related to electron density derivatives: red for strongly repulsive, green for van der Waals, and blue for strongly attractive interactions. Amongst others, $\mathrm{NCl}$ applications cover organic chemistry, ${ }^{[6]}$ inorganic chemistry, ${ }^{[7]}$ biomolecules, ${ }^{[8]}$ solids $^{[9]}$ and crystallography. ${ }^{[10]}$

The original formulation of $\mathrm{NCl}$, although very useful to detect the existence of non-covalent interactions, has only a semiquantitative value. One of the present authors has previously published results where the integration of an ED function over non-covalent regions reproduces rather well the hydrogenbonding potential of selected dimers along the whole energy potential (infinite distance, equilibrium distance and repulsion wall). ${ }^{[11]}$ More recently, G. Saleh et al. ${ }^{[12]}$ have obtained good correlations between complex stabilization energies and kinetic energy densities integrated within reduced density gradient isosurfaces. However, these relations are limited to dimer interactions having a similar physical origin. In a very recent development, ${ }^{[13]}$ considering a frozen electron density (promolecular density), we have proposed a supplementary tool, denoted "IGM" (Independent Gradient Model), which leads to another non-interacting reference. It has been also associated to the ED gradient, leading to the new descriptor $\delta g$ that identifies and quantifies the ED gradient softening due to interaction. Compared to $\mathrm{NCl}$, an attractive feature of this new methodology is to provide a workflow that automatically extracts the signature 
of interactions between selected groups of atoms. In the present paper, we go beyond promolecular densities and we propose a systematic procedure to reveal interaction between chosen atom pairs from an electronic wave function.

Background: The $\mathrm{NCl}$ strategy has been developed to reveal and visualize interactions, based on the troughs that appear in the reduced density gradient $2 \mathrm{D}$ plot $s(\rho)$ (the $\mathrm{NCl}$ descriptor):

$$
s(\rho)=\frac{|\nabla \rho(r)|}{2\left(3 \pi^{2}\right)^{\frac{1}{3}} \rho(r)^{4 / 3}}
$$

This function is used in generalized gradient approximations within the density functional theory to incorporate inhomogeneity corrections to the electron density. ${ }^{[14],[15]}$ The norm $|\nabla \rho|$ of the ED gradient is one critical ingredient of this calculation. On this plot, covalent bonds correspond to troughs occurring at large densities $\rho$. In contrast, drops at low densities reveal weak interactions. Transposing these signals to the real space enables plotting isosurfaces in the cartesian 3D representation, providing chemists with a visual understanding of interactions present in chemical systems.

The $\mathrm{NCl}$ method relies on the use of the electron density $\rho$. Beyond experimental ED, this property can also be addressed by means of quantum chemical calculations (wave function or density functional theory framework). For large chemical systems, such CPU-expensive calculations are rarely feasible. In that case, the promolecular density ${ }^{[16]}$ (sum of simple exponential atomic functions) becomes an attractive option. Lacking ED relaxation, this approximate density has however shown to provide similar results to the relaxed one as far as computations remain in the non-covalent domain..$^{[5]}$ Hence, promolecular densities are useful to study biomolecular systems, typically ligand-receptor interactions, only requiring the geometry as input.

In order to understand the new approach $\delta g$-IGM proposed in this study, it is important to understand where the $s(\rho)$ shape comes from in the $\mathrm{NCl}$ methodology. Without any interaction present in the system, no singular drop appears in the steady exponential decay of the $s(\rho)$ function. That is the reason why any peculiar collapse observed in $s(\rho)$ plays a key role in the success of identifying chemical interactions within the $\mathrm{NCl}$ methodology. However, quantifying these drops within the $\mathrm{NCl}$ approach is difficult due to the need of a non-interacting reference. Initially, monomer densities were used as a reference. However, this reference is not well defined for hetero-dimers, let alone intramolecular interactions. In a recent study, ${ }^{[13]}$ using a promolecular density expression, we looked closer at the origin of such drops observed in the $\mathrm{NCl}$ plot.

In the regions located between two (or more) electron sources (as illustrated at point 1 on the simplified picture provided in Figure 1), the atomic ED contragradience weakens the total gradient $\operatorname{ED}|\nabla \rho|$. It should be noted that Wilson and Goddard first introduced the term contragradience within the molecular orbital approach. They used it for orbital pairs having opposite gradients, ${ }^{[17]}$ which they identified as a characteristic needed for chemical bond formation. As shown in reference ${ }^{[13]}$, this concept

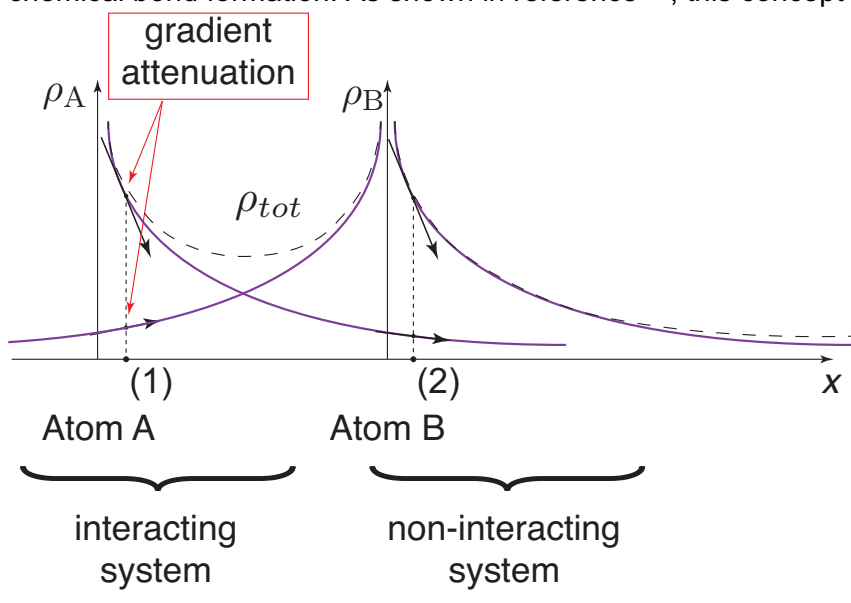

Figure 1. Simplified illustration of the Independent Gradient Model applied fo two electronic sources $A$ and $B$ associated with their own individual ED gradients $\nabla \rho_{A}(r)$ and $\nabla \rho_{B}(r)$ leading to the expression of $\delta \mathrm{g}$ at point $\mathrm{r}$; gradient attenuation occurs at point (1).

can be extended to the ED itself. In the region between electron sources $\mathrm{A}$ and $\mathrm{B}$, since the total gradient $|\nabla \rho|$ is attenuated, falling to zero at the bond critical point (BCP), the numerator of $s(r)$ (and consequently $s(r)$ ) suddenly drops. To quantify this drop, we have previously proposed a new virtual reference characteristic of non-interacting atoms, the independent gradient model (IGM). In this model, promolecular atomic EDs are summed up but the associated atomic gradients are not allowed to interfere. This is achieved by using absolute values upon summing atomic gradients, what erases any ED contragradience feature. This artifice brings the system into a virtual state where individual gradients have been added (like at point 2 on Figure 1 ) but keeping the true ED. The resulting total gradient $\left|\nabla \rho^{I G M}\right|$ is then an upper limit of the true gradient and the difference between them, $\delta g(r)$, quantifies the net ED gradient collapse due to interactions. In other words, the descriptor $\delta g$ identifies the presence of opposite signs in the components of the total ED gradient $|\nabla \rho(r)|$ due to interactions.

The first issue in addressing the IGM implementation is to express the total gradient as a sum of individual terms associated to ED sources. Separating the total ED gradient $\nabla \rho(r)$ into atomic contributions is a natural part of the promolecular (frozen) density. This clearly facilitates the implementation of the IGM model. As the next logical step, the present work aims at extending the range of applicability of the IGM model to relaxed densities calculated from a wave function. This further step towards quantum ED is extremely important to be able to address intra- and intermolecular bonding features in organic chemistry, inorganic and biomolecules examples, with applications in chemical reactivity where large ED rearrangements take place. To this end, in a preliminary step, we propose a well-defined way to cast the orbital-based ED gradient components within the IGM model. To our knowledge, it 
is the first time that such an orbital partitioning of a molecular ED gradient is proposed in view of carrying out an ED topological analysis. A very attractive feature of the IGM model is to provide an uncoupling scheme that automatically separates intra- from inter-fragment interactions. We will show that this possibility remains with ED $\rho$ coming from quantum-mechanical calculations. Finally, a new tool is proposed to extract the signature of atom pair interactions.

The article is organized as follows. In the Methodology section, we first show that a Mulliken-like approach used to divide the ED gradient into orbital contributions is not suitable within the IGM model. Next, using a single-determinant wave function, we propose the Gradient-Based Partition (GBP) scheme that gives relevant results. Then, we present an implementation of the IGM model based on that gradient partitioning. In the Results and Discussion section we show plots of $s(r)$ and $\delta g(r)$ for the water dimer, explaining how the IGM model actually extracts information on interactions present in the chemical system. After interpreting these plots, we provide two prototypical examples of organic chemistry in view of showing the possibilities offered by the two uncoupling schemes proposed (interactions between fragment or atom pairs).

\section{Methodology}

It should first be noted that dividing the total ED $\rho$ into individual sources (orbitals or atoms) is not an imperative requirement to implement the IGM model. Indeed, the aim of the IGM approach is not to derive atomic charges. The opportunity for using the IGM model only relies on the ability to divide the total ED gradient $\nabla \rho$ into local contributions that may be assigned to ED sources. This way, a non-interacting reference (IGM) can be defined in which the individual terms involved in the expression of the total gradient are added up in absolute value. To achieve this, we first show hereafter that a Mulliken-like approach is not suitable. Next, we propose the Gradient-Based Partition (GBP) to be used within the IGM approach.

Mulliken, a partition scheme unsuited to the IGM model: In principle, an obvious strategy to construct a partitioned ED gradient would be to start from a partitioned ED. By nature, the promolecular ED satisfies this condition (sources are atoms), and subsequently its associated gradient does. We took this path in our previous work to derive the $\boldsymbol{\delta} \boldsymbol{g}$ descriptor based on promolecular density. ${ }^{[13]}$ In contrast, ED calculated from a wave function cannot straightforwardly be assigned to individual atoms or orbitals. In the case of a closed-shell system described by a single determinant wave function with each molecular orbitals $\boldsymbol{\psi}_{\boldsymbol{k}}$ (MO) occupied by 2 electrons, the total ED $\boldsymbol{\rho}$ at $\boldsymbol{r}$ is given by:

$$
\rho(r)=2 \sum_{k=1}^{n}\left|\psi_{k}(r)\right|^{2}
$$

The sum is over $\boldsymbol{n}$ spatial normalized MOs (system with $2 \boldsymbol{n}$ electrons). When each $\mathrm{MO} \psi_{k}$ is written as a linear combination of $\mathrm{N}$ normalized atomic orbitals $(\mathrm{AO}) \boldsymbol{\varphi}$, it comes:

$$
\rho(r)=\sum_{i=1}^{N} \sum_{j=1}^{N} D_{i j} \varphi_{i}(r) \varphi_{j}(r)
$$

with $D_{i j}$ a term of the density matrix $\left(D_{i j}=\sum_{k=1}^{n} 2 C_{k i} C_{k j}\right)$.Thus, the partitioning of the ED built up from a wave function calculation is not unique due to the existence of overlap terms $\varphi_{i} \times \varphi_{j}$ that cannot be univocally ascribed to individual orbitals (and subsequently to individual atoms). This problem is well known in quantum chemistry. Many partitioning basis set-based schemes have been proposed in the past to divide up this overlap quantity over orbitals and hence to decompose a molecule in atoms. ${ }^{[18]}$ One of the most known is the Mulliken approach $^{[19]}$ that divides up equally between the overlapping basis functions the integrated electron density coming from the shared electrons. It defines the population $\boldsymbol{N}_{\boldsymbol{A}}$ for an atom $\boldsymbol{A}$ of the molecule as,

$$
N_{A}=\sum_{i \in A} N_{i}
$$

with the sum running over the AOs $i$ centered on atom $A$ and $N_{i}$ the number of electrons assigned to the $\mathrm{AO} \varphi_{i}$ given by,

$$
N_{i}=(D S)_{i, i}=\sum_{j=1}^{N} D_{i j} \int_{\tau} \varphi_{j} \varphi_{i} d \tau
$$

with $\boldsymbol{D}$ and $\boldsymbol{S}$ the density matrix and overlap matrix, respectively. Following Equation (5), it is then tempting to consider the orbital ED local source $\rho_{i}$ associated to the AO $\varphi_{i}$ :

$$
\rho_{i}=\sum_{j=1}^{N} D_{i j} \varphi_{j} \varphi_{i}
$$

The resulting component $x$ of the ED gradient contribution for AO $\varphi_{i}$ would then be:

$$
\frac{\partial \rho_{i}}{\partial x}=\frac{\partial \varphi_{i}}{\partial x} \sum_{j=1}^{N} D_{i j} \varphi_{j}+\varphi_{i} \sum_{j=1}^{N} D_{i j} \frac{\partial \varphi_{j}}{\partial x}
$$

Unfortunately, this ED gradient decomposition scheme leads to undesirable results within the $\boldsymbol{\delta} \boldsymbol{g}$-IGM approach (presented hereafter). Preliminary tests have been performed on the molecule $\boldsymbol{C}_{2} \mathrm{H}_{2}$, including amongst others, unbalanced basis sets (see ESI, Figure S1). It does not describe suitably the interaction regions. This is likely due to the mathematical form of $\partial \rho_{i} / \partial x$ that is not solely governed by AO gradient $\partial \varphi_{i} / \partial x$ but also involves derivatives taken on other AOs $\boldsymbol{\varphi}_{j}$. Moreover, 
results are very sensitive to the basis set, as is known to happen in the calculation of charges. That is the reason why in this paper, this Mulliken partitioning is not used. Instead, we formulate an orbital partition of the ED gradient without resorting to any ED decomposition scheme, what leads to a formula [Eq. (10)], different from Equation (7).

Gradient-Based Partitioning (GBP): Starting from Equation (3), the component $x$ of the ED gradient is given by:

$$
\frac{\partial \rho}{\partial x}=\sum_{i=1}^{N} \sum_{j=1}^{N} D_{i j}\left[\frac{\partial \varphi_{i}}{\partial x} \varphi_{j}+\varphi_{i} \frac{\partial \varphi_{j}}{\partial x}\right]
$$

Since each element $\varphi_{j}\left(\partial \varphi_{i} / \partial x\right)$ appears twice in the symmetric $\boldsymbol{i} \times \boldsymbol{j}$ matrix, we propose to put together the terms with the same orbital derivative (which in the Mulliken scheme would belong to different atomic contributions, see ESI for an example):

$$
\begin{gathered}
\frac{\partial \rho}{\partial x}=\sum_{i=1}^{N} \frac{\partial \rho_{i}}{\partial x} \\
\frac{\partial \rho_{i}}{\partial x}=2 \frac{\partial \varphi_{i}}{\partial x} \sum_{j=1}^{N} D_{i j} \varphi_{j}
\end{gathered}
$$

It is important to note that this gradient partitioning differs from the gradient partitioning derived from Mulliken in Equation (7) (as demonstrated in ESI). Here, although all AOs $\varphi_{j}$ are involved in Equation (10), the derivative is only taken on $\varphi_{i}$. We then propose that each term $\partial \rho_{i} / \partial x$ be considered as an individual gradient element assigned to $\mathrm{AO} \varphi_{i}$ (similar equations apply to components $y$ and $z$ ). Hence, the partition is carried out uniquely for each orbital although the final terms are non-local (they involve all other orbitals). For interpretation purposes, it can be noticed that component $\partial \rho_{i} / \partial x$ can also be expressed as:

$$
\frac{\partial \rho_{i}}{\partial x}=4 \frac{\partial \varphi_{i}}{\partial x} \sum_{k=1}^{n} C_{k i} \psi_{k}
$$

Independent Gradient Model: The presence of individual components of opposite signs in the sum given by Equation (9) causes an attenuation of the total gradient. This is illustrated in Figure 1 in between electron sources $A$ and $B$ (point 1). To quantify the degree of interaction between ED sources with such opposite gradients we need a non-interacting reference. Based on Equation (9), to build this gradient reference, the idea is then to mathematically erase any contragradience feature in this sum. It can be done by artificially imposing the same sign for every term. In our previous study ${ }^{[13]}$ in which we introduced the independent gradient model based on atomic densities (promolecular ED) this was achieved by adding up in absolute value the atomic gradient components in this sum. However, in this case (QM calculations) ED sources are orbitals and it is important to avoid the detection of intra-atomic contragradience. Indeed, in an atom, such possible intershell contragradience does not correspond to interactions between atoms that we want to characterize. In order to do so, electron sources at each point $\boldsymbol{P}$ are divided as $\boldsymbol{L}$ or $\boldsymbol{R}$ depending on their situation with respect to $\boldsymbol{P}$. Hence, the IGM reference at that point is constructed as follows for each orthogonal direction:

$$
\left(\frac{\partial \rho}{\partial x}\right)^{I G M}=\left|\sum_{l=1}^{L} \frac{\partial \rho_{l}}{\partial x}\right|+\left|\sum_{r=1}^{R} \frac{\partial \rho_{r}}{\partial x}\right|
$$

where $\boldsymbol{l}$ runs over all AOs located on the left of grid point $\boldsymbol{P}$, and $\boldsymbol{r}$ runs over all AOs located on the right of grid point $\boldsymbol{P}(\boldsymbol{N}=\boldsymbol{L}+$ $\boldsymbol{R})$. This way, thanks to the use of absolute values, only the interactions between contragradient electron sources of groups $\boldsymbol{L}$ and $\boldsymbol{R}$ are turned off. Therefore, the independent gradient model is a virtual reference with the true ED $\rho$ but in which ED gradient sources facing each other in space are not allowed to interfere. It is worth noting that, following this procedure, AOs centered on the same atom necessarily belong to the same group ( $\boldsymbol{L}$ or $\boldsymbol{R}$ ) and hence won't be allowed to interact within the descriptor $\boldsymbol{\delta} \boldsymbol{g}$ described hereafter.

The norms $|\nabla \rho|$ and $\left|\nabla \rho^{I G M}\right|$ of the true ED gradient and of the reference IGM gradient, respectively, can be computed based on the three components described through Equations (9) and (12), respectively.

Descriptor $\boldsymbol{\delta} \boldsymbol{g}$ within IGM: the new index $\boldsymbol{\delta} \boldsymbol{g}$ defined in our previous work ${ }^{[13]}$ is based on the difference between the noninteracting reference $\left|\nabla \rho^{I G M}\right|$ (the norm of the ED gradient calculated with the IGM model) and the real system:

$$
\delta g=\left|\nabla \rho^{I G M}\right|-|\nabla \rho|
$$

The gradient $\left|\nabla \rho^{I G M}\right|$ being an upper limit of the true molecular ED gradient norm $|\nabla \boldsymbol{\rho}|, \boldsymbol{\delta} \boldsymbol{g}(>\mathbf{0})$ is a measure of the ED gradient collapse caused by electron sharing between contragradient ED sources present in the chemical system. Possibly, we could consider $\delta s=s(\rho)^{I G M}-s(\rho)$ (i.e., $\delta g / \rho^{4 / 3}$ ). It has not been employed in the present work since we demonstrated in our previous work ${ }^{[13]}$ that, due to its mathematical form (not dividing by $\rho^{\mathbf{4} / \mathbf{3}}$ ), $\boldsymbol{\delta} \boldsymbol{g}$ is better suited for the IGM reference. However, for the sake of clarity and to progress toward the understanding of the $\delta \boldsymbol{g}$ content, $\boldsymbol{s}(\boldsymbol{\rho})$ and $\boldsymbol{s}(\boldsymbol{\rho})^{I G M}$ are reported and compared for the first two 1D examples.

On Figure 2a, the first application of the IGM reference to the hydrogen molecule $\boldsymbol{H}_{2}$ is addressed at the quantum-mechanical low level of theory HF/STO-3G. $|\nabla \rho|$ (dashed red line) and $\left|\nabla \rho^{I G M}\right|$ (red circles) exactly coincide outside the inter-nuclear 
region where no ED interference is expected. Between the atoms, the new reference $\left|\nabla \rho^{I G M}\right|$ is always greater than the gradient $|\nabla \rho|$, giving rise to non-zero values of $\delta \boldsymbol{g}$ in this region. As expected, the largest value of $\boldsymbol{\delta} \boldsymbol{g}(0.7$ u.a.) occurs at BCP. The plots $\boldsymbol{s}(\boldsymbol{\rho})$ and $\boldsymbol{s}(\boldsymbol{\rho})^{I G M}$ (obtained with Equation (1) but using reference $\left.\left|\nabla \rho^{I G M}\right|\right)$ ) are also reported. The drop observed between atoms in the $\mathrm{NCl}$ original descriptor $\boldsymbol{s}(\boldsymbol{\rho})$ cancels in the $\boldsymbol{s}(\boldsymbol{\rho})^{I G M}$ representation. It can be noticed that, like the $\mathrm{NCl}$ descriptor, $\boldsymbol{\delta} \boldsymbol{g}$ describes interactions not only at the BCP but also in its surroundings.

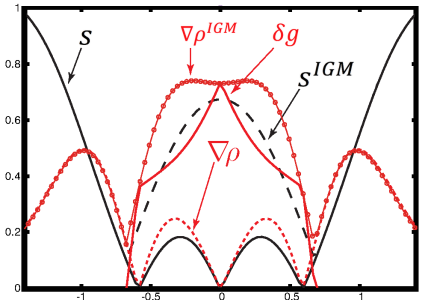

(a) $\mathrm{H}_{1} \quad \mathrm{r}(\mathrm{a} . \mathrm{u}) \quad \mathrm{H}_{2}$

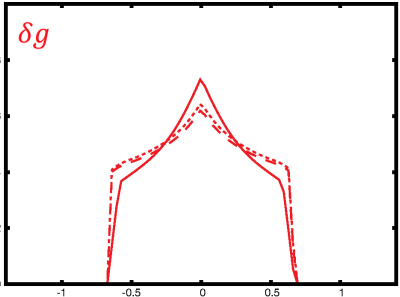

(b) $\mathrm{H}_{1}$

$\mathrm{H}_{2}$
Figure 2. $H_{2}$ molecule (dHH $=1.35$ a.u., obtained at the HF/STO-3G level of theory) (a) $|\nabla \rho|$ (dashed red line), $\left|\nabla \rho^{I G M}\right|$ (red circles) and $\delta g$ (solid red line); $s(\rho)$ (solid black line), $s(\rho)^{I G M}$ (dashed black line); properties calculated from HF/STO-3G wave function along the inter-nuclear axis for $\mathrm{H}_{2}$ with the zero set at the bond critical point; (b) $\delta g$ (a.u.) computed at three different levels of theory: HF/STO-3G (solid line), HF/6-311++G** (with diffuse and polarizations functions on hydrogens, dashed line) and DFT(M06-2X/6$311++G^{\star *}$, dotted line)

The effect of extending the primitive basis set has been investigated using the $6-311++G^{* *}$ basis set (which corresponds to adding diffuse and polarization functions on $\mathrm{H}$ atoms). The two resulting $\boldsymbol{\delta} \boldsymbol{g}$ plots on Figure $2 \mathrm{~b}$ exhibit very similar shapes with a relative small difference in $\boldsymbol{\delta} \boldsymbol{g}$ at BCP (less than 14\%). The use of density functional theory (M06-2X functional) hardly changes the $\boldsymbol{\delta} \boldsymbol{g}$ plot in spite of its known failure to reproduce correct densities. ${ }^{[20]}$ The study of the stability of $\boldsymbol{\delta} \boldsymbol{g}$ versus the level of theory has been extended to the $\boldsymbol{F H} \ldots \boldsymbol{N C H}$ system and reported in ESI. It appears that when the GBP scheme is employed within the IGM approach, the resulting interaction signature (both covalent and non-covalent) measured with $\boldsymbol{\delta} \boldsymbol{g}$ remains nearly unaltered.

In a second step, we have computed the $\boldsymbol{s}(\boldsymbol{\rho}), \boldsymbol{s}(\boldsymbol{\rho})^{I \boldsymbol{G M}}$ and $\boldsymbol{\delta} \boldsymbol{g}$ for the nitrogen molecule. As shown in Figure $3 a, s(\rho)^{I G M}$ detaches from $\boldsymbol{s}(\boldsymbol{\rho})$ in the inter-nuclear region: -0.5 to $0.5 \mathrm{bohr}$, where $\boldsymbol{\delta} \boldsymbol{g}$ takes non-zero values with a maximum at the BCP. Compared to $\boldsymbol{H}_{\mathbf{2}}\left(\boldsymbol{\delta} \boldsymbol{g}_{\max }=0.7\right.$ u.a.), more substantial ED gradient drops are observed in the case of $\boldsymbol{N}_{2}\left(\boldsymbol{\delta} g_{\max }=1.7\right.$ u.a. $)$. This result is in agreement with the respective bond orders, triple in $\mathrm{N}_{2}$ whilst single in $\boldsymbol{H}_{2}$. Next, we have assessed the contribution of each individual molecular orbital $\psi_{k}$ to the descriptor $\boldsymbol{\delta g}$. To do so, in Equation (11), the sum over MOs was reduced to one single term $\boldsymbol{k}$ according to the MO examined. The results are presented in Figure $3 \mathrm{~b}$ for each occupied MO $\boldsymbol{k}$ of $\boldsymbol{N}_{2}$. Although non-additive ( $\boldsymbol{g} \boldsymbol{g}$ is based on a norm calculation and is not he sum of the contributions reported on this figure), valuable information can be drawn from this MO analysis. As can be seen, core orbitals do not participate to the ED gradient collapse occurring between nuclei. In contrast, bonding molecular orbitals $\sigma_{2 s}$ and $\sigma_{2 p z}$ make important individual contribution to $\delta \boldsymbol{g}$. The (filled) anti-bonding orbital $\boldsymbol{\sigma}_{2 s}^{*}$ features absolutely no ED gradient drop in the bonding region. Only small contributions are observed but close to the nucleus. These results can be understood thanks to Equation (11) (see ESI for a full detailed explanation).

A two-dimensional representation of $\boldsymbol{\delta} \boldsymbol{g}$ is given in Figure 3c and $3 \mathrm{~d}$ for MOs $\sigma_{2 p z}$ and $\pi_{y}$, respectively. It offers an illustration of the difference between $\boldsymbol{\sigma}$ - and $\boldsymbol{\pi}$-type bonding in terms of ED gradient softening. While an intense electron sharing is observed along the inter-nuclear axis for $\mathrm{MO} \sigma_{2 p z}$, significant but lower values of $\boldsymbol{\delta} \boldsymbol{g}$ are observed laterally outside the molecular axis for orbital $\boldsymbol{\pi}_{\boldsymbol{y}}$.

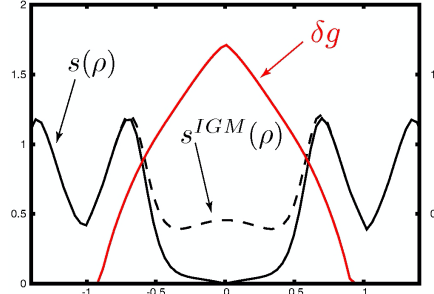

$\mathrm{N}_{1}$

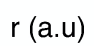

(a)

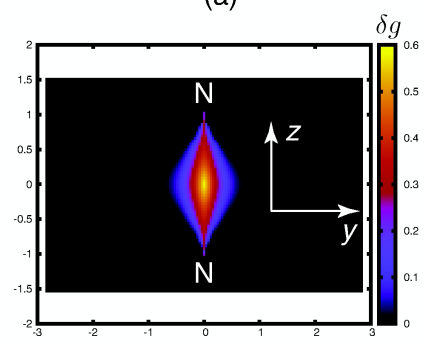

(c)

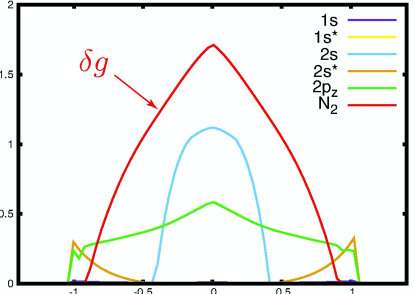

$\mathrm{N}_{2} \mathrm{~N}_{1}$

r (a.u)

(b)

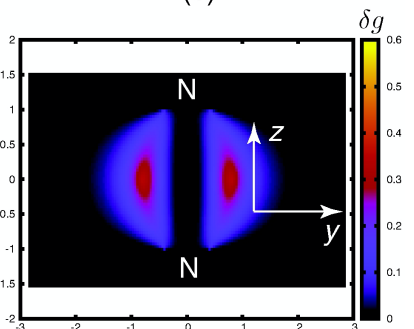

(d)
Figure 3. $N_{2}$ molecule ( $\mathrm{d}_{\mathrm{NN}}=2.1$ u.a.), geometry and wave function obtained at the DFT(M06-2X/6-311++ $\mathrm{G}^{* *}$ ) level of theory (a) $s(\rho)$ (solid black line), $s(\rho)^{I G M}$ (dashed black line) and $\delta g$ (red line); gradients $|\nabla \rho|$ and $\left|\nabla \rho^{I G M}\right|$ are not reported for the sake of clarity; properties calculated along the internuclear axis for $N_{2}$ with the zero set at the bond critical point; (b) $\delta g$ computed individually for 5 occupied MOs of $N_{2}$ (c) $\delta g$ computed in the yOz plane for $\mathrm{MO} \sigma_{2 p z}$ (d) $\delta g$ computed in the yoz plane for MO $\pi_{y}$; properties are reported in a.u..

This result is consistent with the known features of a $\pi$ bond more diffuse than a $\boldsymbol{\sigma}$ bond. Clearly, $\boldsymbol{\delta} \boldsymbol{g}$ detects places where the ED curvature changes upon electron cloud overlapping of atoms.

Comparison to some other descriptors: The underlying idea of the IGM proposal and its associated $\boldsymbol{\delta} \boldsymbol{g}$ descriptor is that 
interaction regions are areas of space where the curvature of the electron density is altered when ED sources interpenetrate. Deviation from the single-exponential behavior normally observed for ED in isolated atoms can be addressed with descriptors such as NCl, LED. ${ }^{[3]}$ Thanks to the use of absolute values, the IGM approach provides a new reference also associated with single-exponential behavior. Just like LED, ${ }^{[21]} \boldsymbol{\delta} \boldsymbol{g}$ belongs to the family of non-dimensionless descriptors.

Automatic separation of intra- and intermolecular interactions: an attractive feature of the independent gradient model is to provide a way to properly cancel the interaction between user selected fragments while preserving interaction inside these fragments. Rearranging Equation 12 like we did in reference ${ }^{[13]}$ we obtain for two molecular fragments $\boldsymbol{A}$ and $\boldsymbol{B}$ :

$$
\left(\frac{\partial \rho}{\partial x}\right)^{I G M, \text { inter }}=\left|\sum_{a=1}^{A} \frac{\partial \rho_{A}}{\partial x}\right|+\left|\sum_{b=1}^{B} \frac{\partial \rho_{B}}{\partial x}\right|
$$

where $\boldsymbol{a}$ runs over all AOs centered on fragment $\boldsymbol{A}$ atoms while $\boldsymbol{b}$ runs over all AOs centered on fragment $\boldsymbol{B}$ atoms. In other words, ED gradient interferences inside each fragment $\boldsymbol{A}$ or $\boldsymbol{B}$ are maintained. But adding the absolute values to the two building blocks $\boldsymbol{A}$ and $\boldsymbol{B}$ turns off any interaction between them. Here, the exponent term "IGM,inter" means that only interactions between fragments are cancelled. This quantity is then used to extract solely the desired $\boldsymbol{A}-\boldsymbol{B}$ interaction present in $|\nabla \rho|$. Accordingly, we define $\delta g^{\text {inter }}$ (and subsequently

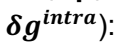

$$
\begin{gathered}
\delta g^{\text {inter }}=\left|\nabla \rho^{I G M, i n t e r}\right|-|\nabla \rho| \\
\delta g^{\text {intra }}=\left|\nabla \rho^{I G M}\right|-\left|\nabla \rho^{I G M, \text { inter }}\right|
\end{gathered}
$$

with these contributions being well separated and thus additive, $\boldsymbol{\delta} \boldsymbol{g}=\boldsymbol{\delta} \boldsymbol{g}^{\text {intra }}+\boldsymbol{\delta} \boldsymbol{g}^{\text {inter }}$. In this uncoupling scheme, $\boldsymbol{\delta} \boldsymbol{g}^{\text {intra }}$ and $\boldsymbol{\delta} \boldsymbol{g}^{\text {inter }}$ separately describe interactions inside each fragment and between fragments, respectively.

Pair interaction: Finally, let us consider Equation (12) limited to the subset of $\boldsymbol{M}$ AOs centered on only two selected atoms:

$$
\left(\frac{\partial \rho}{\partial x}\right)^{I G M, \text { pair }}=\left|\sum_{l=1}^{L_{M}} \frac{\partial \rho_{l}}{\partial x}\right|+\left|\sum_{r=1}^{R_{M}} \frac{\partial \rho_{r}}{\partial x}\right|
$$

with $\boldsymbol{M}=\boldsymbol{L}_{\boldsymbol{M}}+\boldsymbol{R}_{\boldsymbol{M}}$ being the total number of AOs on these two atoms $\left(\boldsymbol{L}_{M}\right.$ and $\boldsymbol{R}_{M}$, the orbitals to the left and right of grid point $\boldsymbol{P}$, respectively). Thanks to the absolute values, the IGM approach provides a reference where ED gradient interferences are cancelled at every point $\boldsymbol{P}$ lying between these two atoms, compared to the situation where gradient attenuation can occur:

$$
\left(\frac{\partial \rho}{\partial x}\right)^{p a i r}=\left|\sum_{m=1}^{M} \frac{\partial \rho_{m}}{\partial x}\right|
$$

(which is the same as Equation (9) but limited to the subset of $\boldsymbol{M}$ AOs centered on the two selected atoms). The resulting $\delta g^{\text {pair }}=\left|\nabla \rho^{\text {IGM,pair }}\right|-\left|\nabla \rho^{\text {pair }}\right|$ will take non-zero values when electronic clouds located on the atom pair will perturb each other.

\section{Results and Discussion}

The water dimer is first selected for testing the $\delta g$ descriptor carried out from an electronic wave function as a prototype of hydrogen bonding. In the following, like in the $\mathrm{NCl}$ methodology, the sign of the second eigenvalue of the ED hessian matrix is used to distinguish between attractive $\left(\lambda_{2}<0\right)$ and repulsive $\left(\lambda_{2}>0\right)$ situations. First, as previously demonstrated with promolecular density, ${ }^{[13]}$ Figure $4 a$ evidences that the ED gradient $|\nabla \rho|$ itself carries the information on the presence of interaction: drops are observed for the gradient in the same place as for $s(\rho)$, i.e. around the density critical points. In the $s(\rho)$ plot, the division by $\rho^{4 / 3}$ magnifies the troughs, mainly at low density where the hydrogen-bond interaction is revealed in a more obvious way than on the $|\nabla \rho|$ plot. In Figure $4 \mathrm{~b}$, all troughs (strong and weak interactions) have been cancelled by using our reference $\left|\nabla \rho^{I G M}\right|$. This result justifies and endorses our IGM approach coupled to the proposed GBP scheme over the whole spectrum of interactions using self-consistent quantummechanical ED. Moreover, the inter/intra-uncoupling IGM scheme [Eqs. 14, 15, 16] delivers the expected result on Figure 4c: we can specifically have access to intramolecular troughs, weak interactions being now fully eliminated from the $\mathrm{NCl}$ plot. $\mathrm{A}$ mere difference in the plots $4 c$ and $4 a$ would then provide the intermolecular interaction. The $\delta g$ descriptor is plotted on Figure $4 \mathrm{~d}$ as a function of the signed ED. Signals observed on both $\mathrm{NCl}$ plots and $\delta g$ plots exactly coincide since they are centered on BCPs. The wide spike revealed at high-density corresponds to $O-H$ covalent bonds whilst hydrogen bonding is disclosed at low-density. With a single treatment, thanks to the IGM uncoupling scheme, detailed information can be directly obtained on the non-covalent domain (Figure 4e). It can be noticed that the hydrogen bond is characterized by small ED gradient alterations $\left(\delta g_{\max }=0.06\right.$ u.a.), one order of magnitude lower than ED interferences detected for the $O-H$ bond $\left(\delta g_{\max }=0.9\right.$ u.a.). Thus, whilst the absolute value of the $\mathrm{NCl}$ descriptor $s(\rho)$ is not characteristic of the kind of interaction, $\delta g$ is clearly able to distinguish between strong and weak interactions. However, we would like to emphasize that $\delta g$ values are in no case connected to interaction energy. $\delta g$ does not consider fragments taken at infinite separation. In other respects, it can be noticed (orange plot on Figure 4f) that promolecular ED clearly underestimates ED gradient drops for 


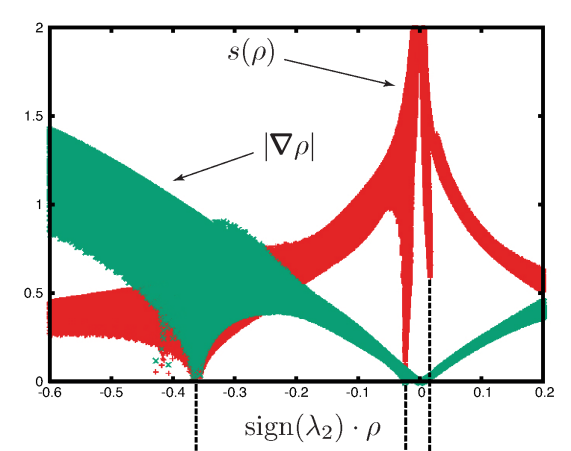

(a)

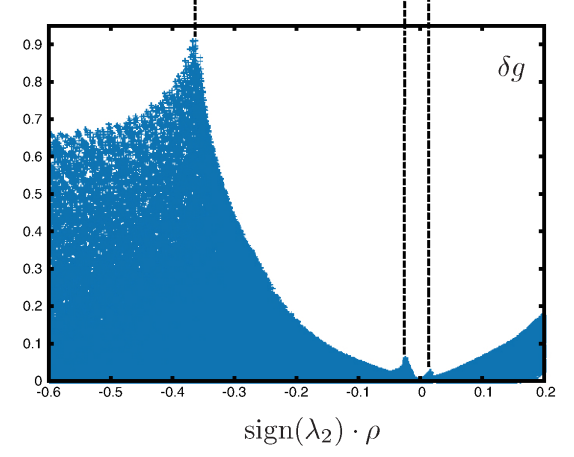

(d)
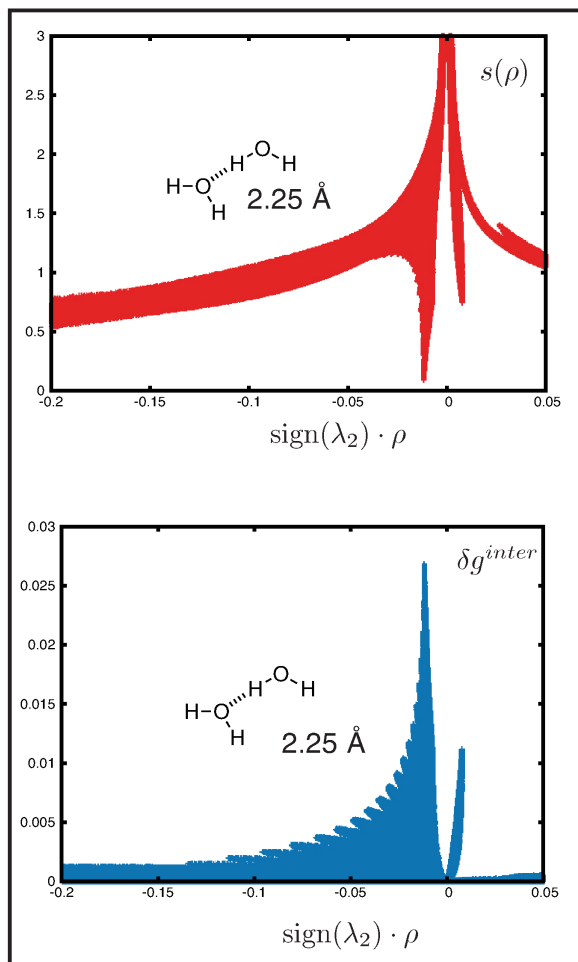

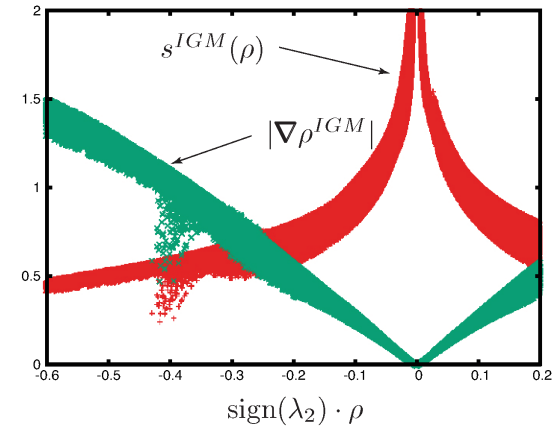

(b)

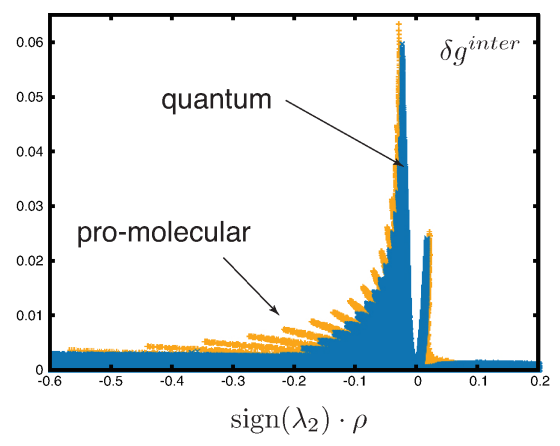

(e)
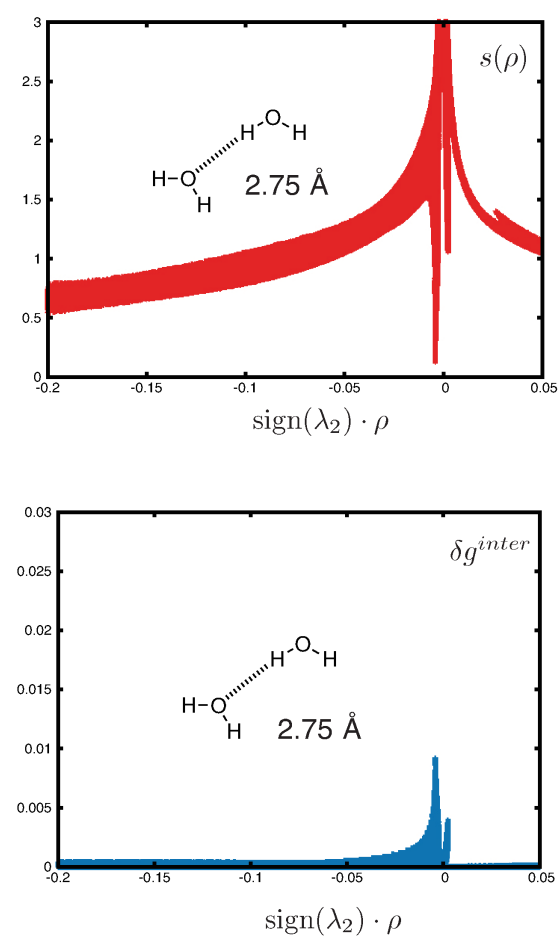

(g)

Figure 4. $s(\rho)$ (red), ED gradient (green), $\delta g(\rho)$ (blue), promolecular $\delta g(\rho)$ (orange); (a) and (d): all interactions are preserved, (b) all interactions cancel, (c) and (f) intermolecular interactions are turned off, (e) intermolecular interactions, $(g)$ evolution of $s(\rho)$ and $\delta g(\rho)$ along the water dimer dissociation reaction path; ED obtained at the DFT(M06-2X/6-311++G**) level of theory; properties reported in a.u.
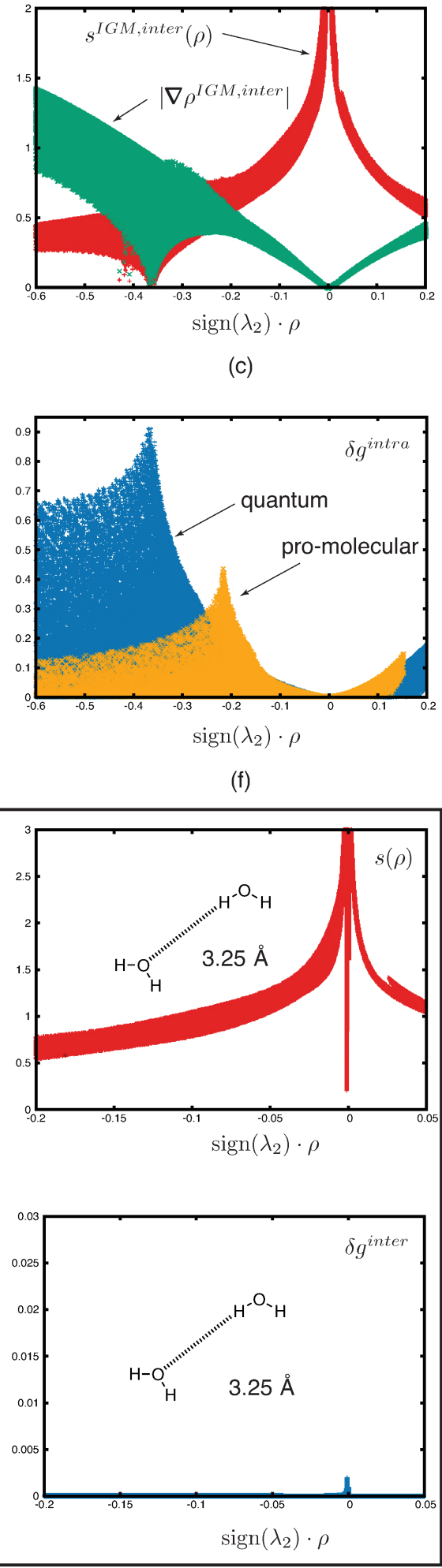

(c)

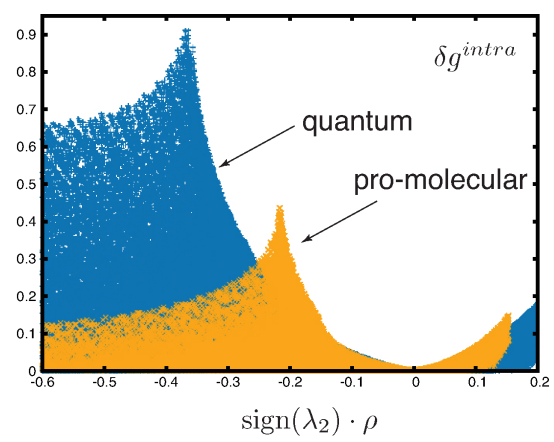

(f)

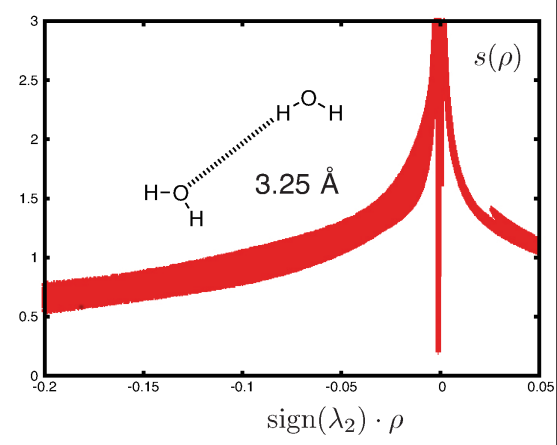


covalent bonding $\left(\delta g_{O-H}^{\text {pro }}=0.4\right.$ versus $\left.\delta g_{O-H}^{Q M}=0.9\right)$. In contrast, in the domain of weak interactions, SCF quantummechanical ED and frozen promolecular ED have very similar features, which has served to validate the promolecular approach for weak interaction in the past. As can be expected from Equation (1), $\delta g(r)$ and $s(r)$ signals coincide on the ED axis as far as Atoms In Molecules critical points are concerned $(s(\rho)$ shows more critical points related to the shell structure). But, one advantage of the $\delta g$ approach against the $\mathrm{NCl}$ method (without a reference) is that peaks are only observed when interactions occur. For instance, the $\delta g^{\text {inter }}$ plot shown in Figure $4 \mathrm{~g}$ has very well delimited peaks with signal gradually vanishing as we stretch the hydrogen bond thanks to the use of the IGM reference. Instead, the $s(\rho)$ representation keeps displaying a sharp spike at very low density due to the symmetry imposed by the presence of a bond critical point, even when there is no interaction anymore. In this case, the analysis of the absence of interaction has to be done in 3D: the volume of the $s(\rho)$ isosurface vanishes, as shown in reference ${ }^{[11]}$.

Let us now turn to the visual analysis in molecular space provided by the $\delta g$ descriptor. In the same fashion as described for $s(\rho), \delta g$ iso-surfaces can be constructed and colored on a BGR color scale according to the density value orientated with the sign of $\lambda_{2}$.

As expected from its own definition, $\delta g$ isosurfaces are centered on the BCP (not directly apparent in Figure 5). The picture delivered by the $\delta g^{\text {intra }}$ descriptor only shows covalent bonds. Compared to the $s(\rho)$ descriptor not dividing by $\rho^{4 / 3}$ in $\delta g$ gives rise to a slightly different shape of the covalent envelopes (more elongated). Also, a more convexshape is obtained for $\delta g^{\text {inter }}$ compared to the compact pillshape of the $s(\rho)$ isosurface.

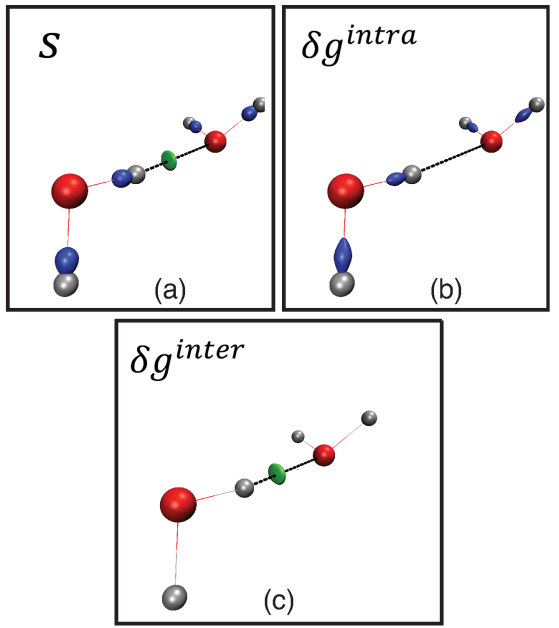

Figure 5. Comparison between interaction isosurfaces for the water dimer (isovalues in a.u.); color coding in the range $-0.4<\operatorname{sign}\left(\lambda_{2}\right)<0.4$ a.u.; (a) $s(\rho)=0.22$, (b) $\delta g^{\text {intra }}=0.67$, (c) $\delta g^{\text {inter }}=0.048$.

Whereas $\mathrm{NCl}$ was developed to specially highlight noncovalent features, this requires the definition of cutoff densities. When IGM is used, applying appropriate ED cutoffs is no longer required to select the desired interaction window (strong intramolecular or weak intermolecular) and to generate associated isocontours. Moreover, the proposed procedure is able to automatically separate weak intra- and intermolecular interactions without handling input parameters. This uncoupling workflow is not limited to the study of intermolecular interactions. Indeed, user-defined fragments can be pieces of a single molecule. The following study of the hexamethylbenzene dication is a noteworthy example of the $\delta g^{\text {inter }}$ usefulness. Very recently, the pentagonal pyramidal structure of this species was confirmed by X-ray crystallography and DFT calculations. ${ }^{[22]}$ In this unconventional structure (unstable at room temperature), the interaction between the apical carbon and the five-membered ring involves a hexa-coordinated carbon atom. The very unusual interaction of this apical carbon has prompted us to investigate the bonding situation in this molecule by means of the $\delta g$-IGM approach.

The skeletal formula used by the authors of this publication is reported in square brackets on Figure $6 \mathrm{a}$. It can be formally thought of as composed of the cations $\mathrm{C}_{5}\left(\mathrm{CH}_{3}\right)_{5}{ }^{+}$and $\mathrm{CH}_{3} \mathrm{C}^{+}$. In order to probe the interactions around the controversial apical carbon, the two fragments considered for carrying out the uncoupling IGM scheme are (i) the apical atom (alone), and (ii) the rest of the molecule. The resulting "intra-fragment" $2 \mathrm{D}$ plot is represented in Figure 6a. It is composed of covalent interaction signatures (panel b) well identified by the QTAIM analysis (panel c). It is worth noting that $\delta g^{\text {intra }}$ also unveils vdW interactions between the five methyl groups (panel d) as well as the signature of the ring closure, that takes the form of a cigar-shape isosurface (but not associated with a critical point here).

More interesting is the $\delta g^{\text {inter }} 2 \mathrm{D}$ plot that shows only two kinds of signals (panel e). As expected, the first one is associated with a standard single $C-C$ bond $\left(\delta g_{B C P}=0.50\right)$ illustrated in panel $f$. The second signal is a nearly symmetric twin peak of opposite values of the $\operatorname{sign}\left(\lambda_{2}\right) \rho$ and is significantly weaker $(\delta g=0.30 / 0.28)$. This smaller signal is associated with the (large) unconventional pyramidal $C-C$ distances experimentally observed in this compound $(1.7 \AA)$. It takes the form of a ring envelope on the $3 D$ representation (panel f). In other words, the ED gradient attenuation due to electron sharing between the apical carbon and $\mathrm{C}_{5}\left(\mathrm{CH}_{3}\right)_{5}{ }^{+}$is in fact delocalized over a ring. A careful analysis shows that, according to the sign of $\lambda_{2}$, these ED gradient drops occur in alternating sequence of attractive and repulsive areas. This is fully in line with the QTAIM analysis that discloses alternating bond and ring critical points along a ring located above the $C_{5}$ plane. The BCPs located on the five lines joining the apical carbon to the basal carbons correspond to the attractive part of the $\delta g^{\text {inter }}$ signal. The repulsive part is associated with the five RCPs located between these lines. Looking closer at the $3 \mathrm{D}$ representation (panel $\mathrm{f}$ ), it appears that electron sharing assigned to attractive interaction along the five lines of the pyramid roof ( $\delta g=0.30$ ) expands over a slightly greater volume than the one occurring in repulsive areas $(\delta g=0.28)$. These two types of interaction counterbalance, the net effect being slightly stabilizing and responsible of the (weak) cohesion observed experimentally. 


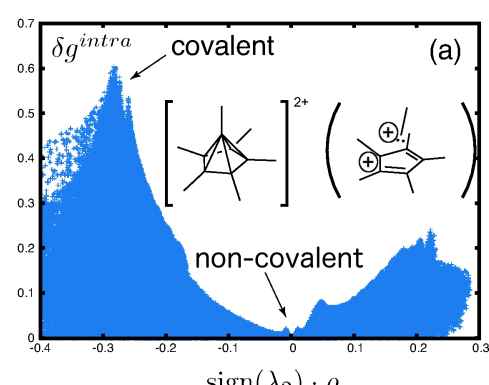

$\operatorname{sign}\left(\lambda_{2}\right) \cdot \rho$

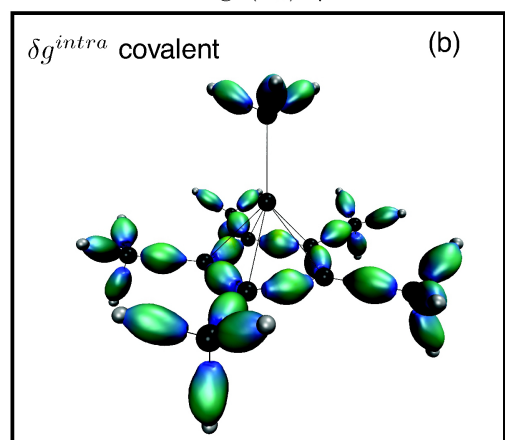

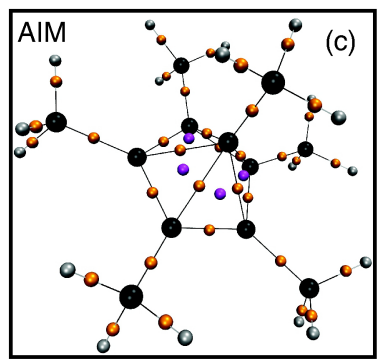

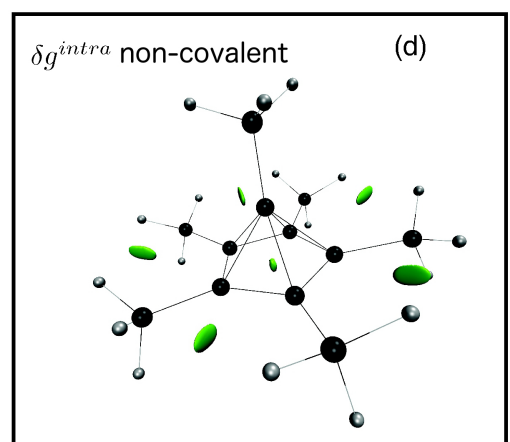

Figure 6. $\delta \mathrm{g} 2 \mathrm{D}$ plots and $3 \mathrm{D}$ isocontours for the hexamethylbenzene dication treated as the assembly of two fragments: apical carbon and the rest of the molecule, color coding in the range $-0.5<\operatorname{sign}\left(\lambda_{2}\right)<0.5$ a.u, isovalues in a.u.; (a), (b) and (d): intra-fragment contribution to $\delta g$ for the two fragments, $\delta \mathrm{g}^{\text {intra/strong }}(\rho)=0.25$ and $\delta \mathrm{g}^{\text {intra/weak }}(\rho)=0.075$ isosurfaces; (c) QTAIM analysis with BCPs and RCPs colored orange and purple, respectively; (e) and (f): inter-fragment contribution to $\delta \mathrm{g}$ for the two fragments, $\delta \mathrm{g}^{\text {inter }}(\rho)=0.25$ isosurface.

Finally, an extremely appealing feature of the IGM approach is the opportunity to monitor separately selected bonds. We have taken the $\beta$-thioaminoacrolein as an example. Its skeletal formula is reported on Figure $7 \mathrm{a}$. As usual, the $\mathrm{NCl}$ analysis discloses troughs in the $s(\rho)$ diagram in the high ED domain (beyond -0.2 u.a.) corresponding to covalent interactions, as well as two sharp signals in the low ED domain, leading to the $3 \mathrm{D}$ representation reported in the same panel. Like $\mathrm{NCl}$, the $\delta g$-IGM approach (panel $7 \mathrm{~b}$ ) is able to describe covalent bonding resulting in isosurfaces of different shapes, which enables to identify bonding type. Compared with the $\delta g$ representations, it is clear that the division by $\rho^{4 / 3}$ used in $\mathrm{NCl}$ stretches the $s(\rho)$ signals on the $2 \mathrm{D}$ plot allowing to capture the whole range of interactions at once (strong and weak including $H \ldots N$ hydrogen-bonding and intramolecular ring-closure) for a given $s(\rho)$ isovalue, what is an advantage over the $\delta g$ approach. But, a further detailed $\delta g$ picture can be obtained thanks to the use of $\delta g^{\text {pair }}$ descriptors. On Figure $7 \mathrm{c}$, we have superimposed $\delta g^{\text {pair }}$ plots computed for 8 atom pairs of the molecule [Eqs. 17 and 18] and associated with covalent bonding or $\mathrm{H}$-bond situations. This treatment reveals the details behind the overall contour of $\delta g(\rho)$, and by extension behind the $\mathrm{NCl}$ profile. In particular, the $C-C$ (green) and $C-H$ (red) peaks clearly emerge from this picture. Also, the $C=C$ peak, initially drown out by the $N-H$ signal is now fully visible. As a consequence, isosurfaces can be separately constructed for pair interactions in the 3D representation. This paves the way to monitor specific interactions occurring during a reaction. Similar to what was previously done within the $\mathrm{NCl}$ approach, ${ }^{[23]}$ we can for instance consider monitoring a $C-X$ breaking bond that progressively moves to a non-covalent situation in the course of a $\mathrm{S}_{\mathrm{N}} 2$ reaction. This work is under progress in our group.

The reader could be tempted to associate $\delta g$ magnitude with the interaction "strength". However, at the present stage of investigation, a deeper understanding of the chemical meaning of $\delta g$ has still to be achieved. The value of $\delta g$ is related with density reconstruction and thus with the strength of the bond, but not in a direct manner. Interaction signatures $\delta g^{\text {pair }}$ have a more or less flared shape suggesting that attempts to correlate energetics or bond indexes to the $\delta \mathrm{g}$ descriptor should consider integration schemes. It is worth noting that $\delta g$-IGM offers a clear advantage over the previous integration scheme (requiring a spline interpolation for the non-interacting system carried out separately) implemented in the $\mathrm{NCl}$ method. ${ }^{[11]}$ Indeed, thanks to the IGM approach, the non-interacting reference can be defined just with an extra coding line, which considerably fastens the calculation. For heterodimers, the issue of choosing between two reference splines no longer arises.

\section{Summary and future outlook.}

This paper reports on an ongoing effort to develop methods able to reveal molecular interaction signatures from the electron density (ED) topology. It follows up a very recent work presenting a new reference model, the independent gradient model (IGM), initially developed for promolecular ED. This model is hereby extended to relaxed ED for closed-shell single determinant wave functions. First, we have casted the orbital-based ED gradient in a form that is most effective to 

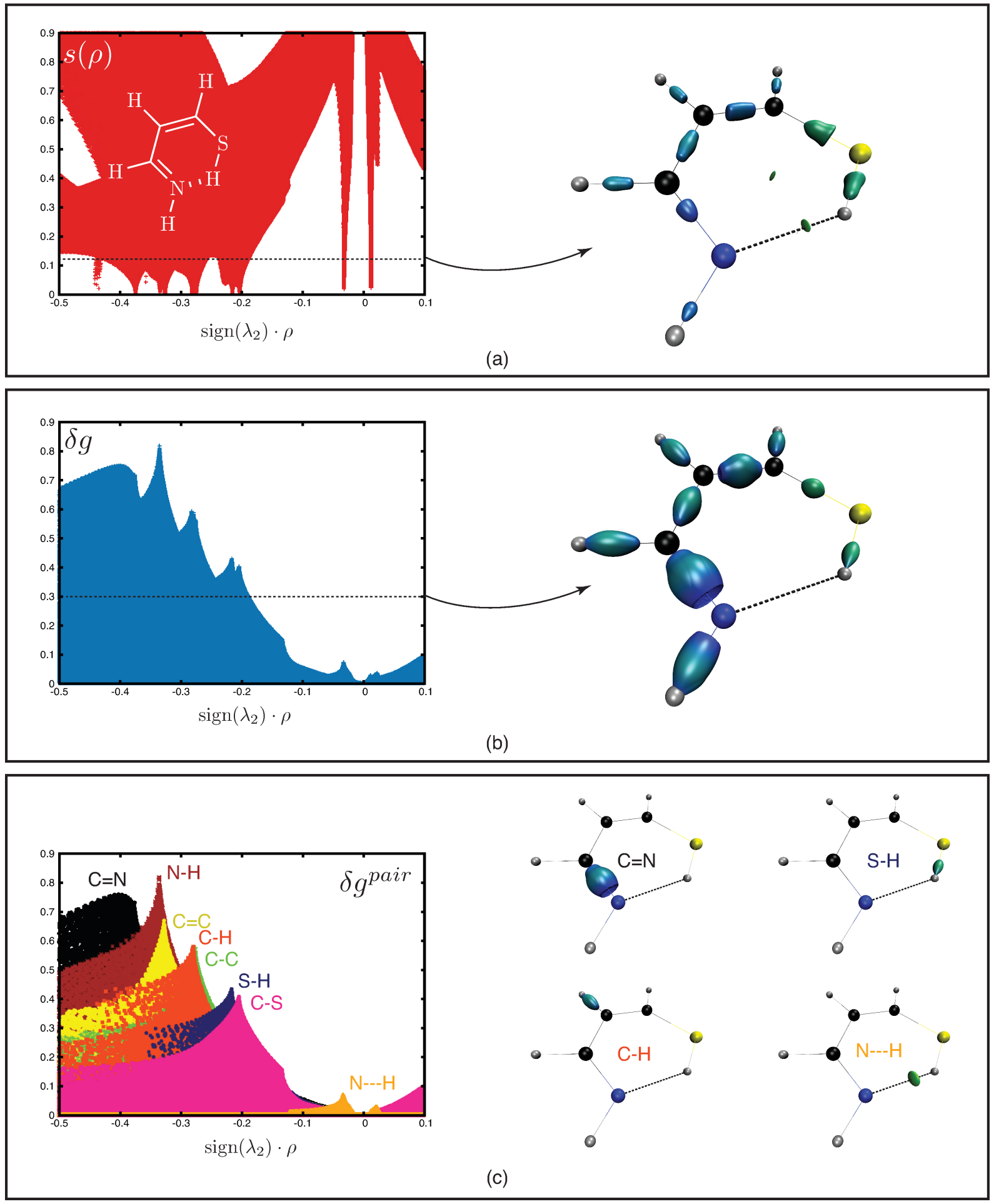

Figure 7. $s(\rho)$ and $\delta g$ 2D and 3D plots for $\beta$-thioaminoacrolein, isosurface color coding in the range $-0.5<\operatorname{sign}\left(\lambda_{2}\right)<0.5$ a.u, isovalues in a.u.; (a) $s(\rho)=0.12$ isosurfaces, (b) $\delta \mathrm{g}=0.30$ isosurfaces, (c) superimposition of 8 individual $\delta \mathrm{g}^{\text {pair }} 2 \mathrm{D}$ plots, selection of three $\delta \mathrm{g}^{\text {pair }}=0.30$ isosurfaces for covalent bonds and one $\delta \mathrm{g}^{\text {pair }}=0.05$ isosurface for the intramolecular $\mathrm{H}$-bond. 
divide it into components that may be assigned to ED gradient sources (atomic orbitals). This Gradient-Based Partitioning (GBP) is the fundamental prerequisite to carry out the IGM approach. Next, regrouping gradient sources in two sets according to their position relative to the current grid point and using absolute values taken around each set, we have proposed a fully non-interacting gradient reference $\left|\nabla \rho^{I G M}\right|$, an upper limit of the true ED gradient $|\nabla \rho|$. The resulting difference, the $\delta g$ descriptor, is a measure of electron sharing brought by ED contragradience, a concept initially developed for orbital pairs having opposite gradients. The model has been analyzed and tested from simple cases to more sophisticated ones. As with the $\mathrm{NCl}$ approach, 3D isosurfaces can be constructed enabling spatial representations covering a large range of interactions associated or not with bond critical point: covalent bond, hydrogen bonds, van der Waals interactions and steric clashes. One major interest of $\delta g$ is to only describe interaction situations making it a key asset for integration schemes that have emerged in the past few years to find a relationship between local properties in such regions and energetics properties. It is however to be noted that, by construction, the $\delta g$-IGM approach is limited to theoretical ED models, ruling out the use of experimental ED, whilst the $\mathrm{NCl}$ approach has not such limitation. Another advantage of these $s(\rho)$ isosurfaces is that they lead to "chemical" topological charges" connected to the conceptual meaning of shell structure in atoms. ${ }^{[24]}$

A compelling feature of the IGM model is to enable designing customized ED references cancelling interactions between user-selected groups of atoms. Across two case studies we have illustrated the usefulness of two such IGM schemes. On one hand, we have shown how to uncouple intra- from interfragment interactions. The $\delta g^{\text {inter }}$ index enables the automatic extraction of inter-fragment interaction signatures without supplying any user-dependent parameter. On the other hand, we have made use of a new procedure to specifically target a given atom pair interaction in a molecule. These uncoupling tools should prove useful in exploring the interaction patterns of molecular systems over a large scale of strength. Also they provide new opportunities to monitor chemical changes occurring in a reaction by using data from reaction paths calculations. This work is in progress in our group.

Attempts are currently undertaken to exploit the $\delta g$ descriptor within integration schemes for chemical bonding studies. In fact, although the spatial representations generated from $\delta g$ reflects the common chemical concept of bond in a clear and intuitive manner, being distinctly different for single or double bonds, and although the $\delta g$ magnitude is able to distinguish between weak and strong interactions and even to make an appropriate distinction between certain covalent bonds, the chemical content of this descriptor remains to be determined. Thus, work is still required to establish a proper correlation with properties like energy bonding or force constant, or bonding index.

\section{Computational methods}

All descriptor computations were carried out with IGMPlot [25] (promolecular ED) and a modified version of NCIPlot (quantum mechanics ED). ${ }^{[6]}$ If not otherwise stated, full geometry optimizations have been performed at the DFT (M06-2X/6-311++G**) level of theory in gas phase using the package Gaussian 09. ${ }^{[27]}$ Every minimum was carefully characterized through harmonic analysis (stationary points with no imaginary frequencies). The geometry optimization of the hexamethyl dication has been carried out at the DFT (B3LYP/Def2TZVP) level of theory (singlet state) as mentioned by the authors. ${ }^{[22]}$

\section{Acknowledgements}

We are grateful to the MaSCA (Maison de la Simulation de Champagne-Ardenne, France, http://romeo.univ-reims.fr) and the CRIANN computational center (http://www.criann.fr) for computing facilities. We would like to thank Lucie Wanlin warmly for her help in creating the TOC graphic. Finally, we are grateful to $\mathrm{Pr}$ Frédéric Bohr for helpful discussions on quantum mechanical models.

Keywords: $\mathrm{NCl} \cdot \mathrm{IGM} \cdot$ interaction $\bullet$ QTAIM $・$ descriptor • electron density $\cdot$ non-covalent

[1] a) R. F. W. Bader, Atoms in Molecules: a Quantum Theory, Oxford University Press, 1994, p. 458; b) C. F. Matta, R. J. Boyd in An Introduction to the Quantum Theory of Atoms in Molecules, WileyVCH Verlag GmbH \& Co. KGaA, 2007, pp. 1-34

[2] B. Silvi, A. Savin, Nature 1994, 371, 683-686.

[3] H. J. Bohórquez, C. F. Matta, R. J. Boyd, International Journal of Quantum Chemistry 2010, 110, 2418-2425.

[4] E. R. Johnson, S. Keinan, P. Mori-Sánchez, J. Contreras-García, A. J. Cohen, W. Yang, Journal of the American Chemical Society 2010 132, 6498-6506.

[5] C. Narth, Z. Maroun, R. A. Boto, R. Chaudret, M.-L. Bonnet, J.-P. Piquemal, J. Contreras-Garca in A Complete NCl perspective: From New Bonds to Reactivity, Eds.: R. Chauvin, C. Lepetit, B. Silvi and E. Alikhani), Springer, 2016, pp. 491-527.

[6] A. Armstrong, R. A. Boto, P. Dingwall, J. Contreras-Garcia, M. J. Harvey, N. J. Mason, H. S. Rzepa, Chemical Science 2014, 5 , 2057-2071.

[7] a) J. P. Cerón-Carrasco, J. Cerezo, A. Requena, J. Zuñiga, J. Contreras-García, S. Chavan, M. Manrubia-Cobo, H. PérezSánchez, Journal of Molecular Modeling 2014, 20, 2401; b) C. Werlé, C. Bailly, L. Karmazin-Brelot, X.-F. Le Goff, M. Pfeffer, J.-P. Djukic, Angewandte Chemie 2014, 126, 9985-9989.

[8] a) X. Laurent, N. Renault, A. Farce, P. Chavatte, E. Hénon, PLOS Computational Biology 2014, 10, 1-15; b) J. Gonzalez, I. Baños, I. 
león, J. Contreras-García, E. Cocinero, A. Lesarri, J. Fernández, J. Millán, Unravelling Protein-DNA Interactions at Molecular Level: $A$ DFT and NCI Study, 2016, p.

[9] J. A. Sans, F. J. Manjón, C. Popescu, V. P. Cuenca-Gotor, O. Gomis, A. Muñoz, P. Rodríguez-Hernández, J. Contreras-García, J. PellicerPorres, A. L. J. Pereira, D. Santamaría-Pérez, A. Segura, Physical Review B 2016, 93, 054102.

[10] a) G. Saleh, C. Gatti , L. Lo Presti, J. Contreras-García, Chemistry - A European Journal 2012, 18, 15523-15536; b) A. Otero-de-la-Roza, E. R. Johnson, J. Contreras-Garcia, Physical Chemistry Chemical Physics 2012, 14, 12165-12172.

[11] J. Contreras-García, W. Yang, E. R. Johnson, The Journal of Physical Chemistry A 2011, 115, 12983-12990.

[12] G. Saleh, C. Gatti, L. Lo Presti, Computational and Theoretical Chemistry 2015, 1053, 53-59.

[13] C. Lefebvre, G. Rubez, H. Khartabil, J.-C. Boisson, J. ContrerasGarcia, E. Henon, Physical Chemistry Chemical Physics 2017, 19, 17928-17936

[14] J. M. del Campo, J. L. Gázquez, R. J. Alvarez-Mendez, A. Vela, International Journal of Quantum Chemistry 2012, 112, 3594-3598.

[15] A. Zupan, J. P. Perdew, K. Burke, M. Causà, International Journal of Quantum Chemistry 1997, 61, 835-845.

[16] M. A. Spackman, E. N. Maslen, The Journal of Physical Chemistry 1986, 90, 2020-2027.

[17] C. Woodrow Wilson, W. A. Goddard, Chemical Physics Letters 1970, 5, 45-49.

[18] S. Saha, R. K. Roy, P. W. Ayers, International Journal of Quantum Chemistry 2009, 109, 1790-1806.

[19] R. S. Mulliken, The Journal of Chemical Physics 1955, 23, 1833-1840

[20] M. G. Medvedev, I. S. Bushmarinov, J. Sun, J. P. Perdew, K. A. Lyssenko, Science 2017, 355, 49.

[21] H. J. Bohórquez, R. J. Boyd, Theoretical Chemistry Accounts 2010, 127, 393-400.

[22] M. Malischewski, K. Seppelt, Angewandte Chemie International Edition 2017, 56, 368-370.

[23] N. Gillet, R. Chaudret, J. Contreras-García, W. Yang, B. Silvi, J.-P. Piquemal, Journal of Chemical Theory and Computation 2012, 8, 3993-3997.

[24] K. Wagner, M. Kohout, Theoretical Chemistry Accounts 2011, 128, 3946.

[25] E. Pluot, G. Rubez, J.-C. Boisson, E. Hénon, IGMPlot, 2017. http://kisthelp.univ-reims.fr/IGMPLOT.

[26] J. Contreras-García, E. R. Johnson, S. Keinan, R. Chaudret, J.-P. Piquemal, D. N. Beratan, W. Yang, Journal of Chemical Theory and Computation 2011, 7, 625-632.

[27] M. J. Frisch, G. W. Trucks, H. B. Schlegel, G. E. Scuseria, M. A. Robb, J. R. Cheeseman, G. Scalmani, V. Barone, B. Mennucci, G. A. Petersson, H. Nakatsuji, M. Caricato, X. Li, H. P. Hratchian, A. F. Izmaylov, J. Bloino, G. Zheng, J. L. Sonnenberg, M. Hada, M. Ehara, K. Toyota, R. Fukuda, J. Hasegawa, M. Ishida, T. Nakajima, Y. Honda, O. Kitao, H. Nakai, T. Vreven, J. Montgomery, J. A., J. E. Peralta, F. Ogliaro, M. Bearpark, J. J. Heyd, E. Brothers, K. N. Kudin, V. N. Staroverov, R. Kobayashi, J. Normand, K. Raghavachari, A. Rendell, J. C. Burant, S. S. Iyengar, J. Tomasi, M. Cossi, N. Rega, J. M. Millam, M. Klene, J. E. Knox, J. B. Cross, V. Bakken, C. Adamo, J. Jaramillo, R. Gomperts, R. E. Stratmann, O. Yazyev, A. J. Austin, R. Cammi, C. Pomelli, J. W. Ochterski, R. L. Martin, K. Morokuma, V. G. Zakrzewski, G. A. Voth, P. Salvador, J. J. Dannenberg, S. Dapprich, A. D. Daniels, Ö. Farkas, J. B. Foresman, J. V. Ortiz, J. Cioslowski, D. J. Fox in Gaussian 09 Revision A.1, Vol. Gaussian, Inc., , Wallingford, 2009. 


\section{Entry for the Table of Contents}

\section{FULL PAPER}

The new $\delta g$-IGM approach, hereby extended to electron density obtained from quantum mechanics, is able to provide chemists with a visual understanding of interactions by specifically probing a given atom pair in a molecule. It paves the way for targeted mechanistic exploration of reactions.

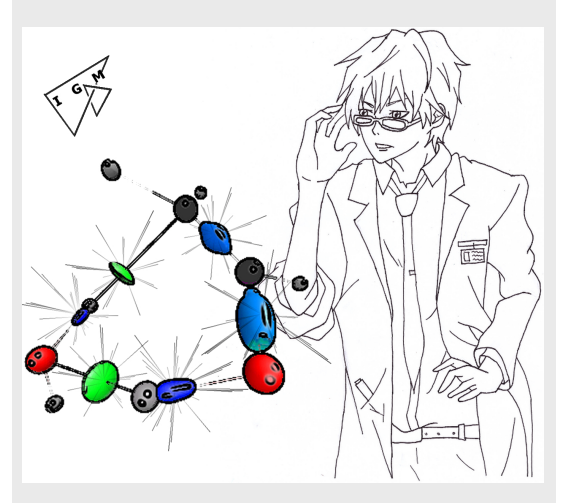

Author(s), Corresponding Author(s)*

Page No. - Page No.

Title 